\title{
QUASI-INVERSE ENDOMORPHISMS
}

\author{
A. CARANTI
}

\begin{abstract}
Greither and Pareigis have established a connection between Hopf Galois structures on a Galois extension $L / K$ with Galois group $G$, and the regular subgroups of the group of permutations on $G$, which are normalized by $G$. Byott has rephrased this connection in terms of certain equivalence classes of injective morphisms of $G$ into the holomorphs of the groups $N$ with the same cardinality of $G$.

Childs and Corradino have used this theory to construct such Hopf Galois structures, starting from fixed-point-free endomorphisms of $G$ that have abelian images. In this paper we show that a fixed-point-free endomorphism has an abelian image if and only if there is another endomorphism that is its inverse with respect to the circle operation in the near-ring of maps on $G$, and give a fairly explicit recipe for constructing all such endomorphisms.
\end{abstract}

\section{INTRODUCTION}

Our starting point is the paper Chi12, in which Childs begins by reviewing the theory of Greither and Pareigis [GP87. This theory establishes a bijection between Hopf Galois structures on a Galois extension $L / K$ of fields with Galois group $G$, and the regular subgroups of the group of permutations on $G$, which are normalized by $G$. Byott By96a, By96b has shown that the problem of determining these regular subgroups can be translated into that of finding certain equivalence classes of injective morphisms of $G$ into the holomorphs of the groups $N$ with the same cardinality of $G$. Childs and Corradino [CC07] and Childs [Chi07] have showed that abelian fixed-point-free endomorphisms of $G$ yield directly, via the above theory, such Hopf Galois structures. (Here we say with Chi12 that an endomorphism is abelian if its image is abelian.) We refer to [Chi12] and [Chi00] for the details.

Childs studies in particular those Hopf Galois structures that arise from the abelian fixed-point-free endomorphism $\varphi$ of $G$ that admit an inverse endomorphism $\psi$ with respect to the circle operation $\varphi \circ \psi=\psi-\varphi \psi+\varphi$. (Childs calls $\psi$ the inverse of $\varphi$. Since he only considers fixed-point-free endomorphisms that are not automorphisms, the term is unambiguous in his context. In this paper we will have to deal also with fixed-point-free automorphisms, and with inverses with respect to map composition. We have thus preferred to use the classical term quasi-inverse for $\psi$ [Jac64, Chap. 1, Section. 5].)

Date: 14 December 2012, 11:29 CET — Version 5.01.

2010 Mathematics Subject Classification. 20D45 16N20 16Y30.

Key words and phrases. Hopf Galois structures, finite groups, fixed-point-free endomorphisms, quasi-inverse, near-ring. 
In this paper we study the fixed-point-free endomorphisms of a finite group that have a quasi-inverse. We will show that for a fixed-point-free endomorphism of a finite group the properties of being abelian, and that of having a quasiinverse, are equivalent (Theorem [3.4, which extends [Chi12, Remark 10], and shows that the condition of having a quasi-inverse is not restrictive in the context of [Chi12]). In Sections 4 and 7 we are able to give reasonably explicit recipes for constructing the groups that have such an endomorphism, and for determining all of their endomorphisms with this property. Our main tool is a version for groups of Fitting's Lemma for modules (Section 44). We are then led to study fixedpoint-free endomorphisms of finite abelian groups (Section 5), abelian nilpotent endomorphisms (Section [6), and how to put the two together (Section (7). In Section 8 we will be reviewing the examples of [Chi12] from our point of view, and provide some more.

\section{Preliminaries}

Let $G$ be a group, multiplicatively written. In our context, it is convenient to write maps on $G$ as exponents. Given two maps $\varphi, \psi$ on $G$, we define as usual their pointwise sum $\varphi+\psi$ by $x^{\varphi+\psi}=x^{\varphi} x^{\psi}$, for $x \in G$ and their product $\varphi \psi$ as their composition, $x^{\varphi \psi}=\left(x^{\varphi}\right)^{\psi}$. It is well known that these operations define a structure of a near-ring on the set $N(G)$ of maps on $G$. (See [Pil83.) The sum is not commutative in general, and only the distributive relation $\varphi(\psi+\vartheta)=\varphi \psi+\varphi \vartheta$ holds generally, while if $\varphi$ is an endomorphism of $G$, we also have $(\psi+\vartheta) \varphi=$ $\psi \varphi+\vartheta \varphi$. Note also that for a map $\varphi$ on $G,-\varphi$ is defined by $\varphi+(-\varphi)=0$ (where $x^{0}=1$ for all $\left.x \in G\right)$, so that $x^{-\varphi}=\left(x^{\varphi}\right)^{-1}$ for $x \in G$. Thus $-(\varphi+\psi)=-\psi-\varphi$ for maps $\varphi, \psi$ on $G$.

If $\varphi, \psi$ are endomorphisms of $G$, their sum $\varphi+\psi$ is not in general an endomorphism of $G$. We have

Proposition 2.1. Let $G$ be a group, $\varphi, \psi \in \operatorname{End}(G)$. The following are equivalent

(1) $\varphi+\psi \in \operatorname{End}(G)$, and

(2) $\left[G^{\varphi}, G^{\psi}\right]=1$, that is, the images of $\varphi$ and $\psi$ commute elementwise.

Moreover, these conditions imply $\varphi+\psi=\psi+\varphi$.

Proof. $(a b)^{\varphi+\psi}=(a b)^{\varphi}(a b)^{\psi}=a^{\varphi} b^{\varphi} a^{\psi} b^{\psi}$ equals $a^{\varphi+\psi} b^{\varphi+\psi}=a^{\varphi} a^{\psi} b^{\varphi} b^{\psi}$ for all $a, b \in G$ if and only if $b^{\varphi} a^{\psi}=a^{\psi} b^{\varphi}$ for all $a, b \in G$. Setting $b=a$ in the last identity we obtain $\varphi+\psi=\psi+\varphi$.

Definition 2.2. Let $\varphi$ be an endomorphism of the group G. A fixed point of $\varphi$ is an element $x \in G$ such that $x^{\varphi}=x$. We say that $\varphi$ is fixed-point-free if its only fixed point is 1 .

The following is well-known Gor68, Lemma 1.1., Chap. 10].

Proposition 2.3. Let $G$ be a finite group, $\varphi \in \operatorname{End}(G)$. The following are equivalent:

(1) the map $1-\varphi$ is injective, and thus a bijection on $G$, and

(2) $\varphi$ is fixed-point-free. 
Proof. $x^{1-\varphi}=x x^{-\varphi}=y y^{-\varphi}=y^{1-\psi}$ if and only if $y^{-1} x=\left(y^{-1} x\right)^{\varphi}$, that is, $y^{-1} x$ is a fixed point of $\varphi$.

\section{QUASI-INVERSE ENDOMORPHISMS}

Childs studies in Chi12 the fixed-point-free endomorphisms $\varphi$ such that $1-\varphi$ has an inverse of the same form $1-\psi$, for some endomorphism $\psi$ of $G$.

Definition 3.1. Let $G$ be a finite group. A fixed-point-free endomorphisms $\varphi$ of $G$ is said to be quasi-invertible if there is an endomorphism $\psi$ of $G$ such that the map $1-\psi$ is the inverse of the map $1-\varphi$ with respect to map composition. $\psi$ is said to be the quasi-inverse of $\varphi$.

Clearly $\psi$ is also fixed-point-free by Proposition 2.3 , and $\psi$ is uniquely determined by $\varphi$. A familiar argument shows that if $\psi$ is the quasi-inverse of $\varphi$, then $1=(1-\varphi)(1-\psi)=1-\varphi+\varphi \psi-\psi$, so that $-\varphi+\varphi \psi-\psi=0$, or

$$
\varphi+\psi=\varphi \psi \text {. }
$$

In other words, $\psi$ is the inverse of $\varphi$ with respect to the circle operation $\varphi \circ \psi=$ $\psi-\varphi \psi+\varphi$ on the near-ring $N(G)$. Thus $\varphi$ is right quasi-regular in the classical radical theory of Jacobson [Jac64, Chap. 1, Section. 5], the catch here being that we require the (right) quasi-inverse $\psi$ of $\varphi$ to be another endomorphism of $G$.

Since $\varphi+\psi=\varphi \psi \in \operatorname{End}(G)$, we have by Proposition $2.1 \varphi+\psi=\psi+\varphi$, and thus $\varphi \psi=\psi \varphi$. Also, $\left[G^{\varphi}, G^{\psi}\right]=1$. Now we can rewrite (3.1) as

$$
\varphi=-\psi+\varphi \psi=(-1+\varphi) \psi
$$

as $\psi \in \operatorname{End}(G)$. This shows that $G^{\varphi} \subseteq G^{\psi}$ and then by symmetry (or because $-1+\varphi$ is a bijection) $G^{\varphi}=G^{\psi}$. Also, if $x \in \operatorname{ker}(\varphi)$, then $1=x^{\varphi}=x^{(-1+\varphi) \psi}=$ $\left(x^{-1}\right)^{\psi}$, so that $x \in \operatorname{ker}(\psi)$, and thus $\operatorname{ker}(\varphi)=\operatorname{ker}(\psi)$. We have obtained

Proposition 3.2. Let $G$ be a finite group, $\varphi, \psi$ two fixed-point-free endomorphisms of $G$ that are one the quasi-inverse of the other. Then

(1) $\varphi+\psi=\psi+\varphi$ and $\varphi \psi=\psi \varphi$,

(2) $G^{\varphi}=G^{\psi}$ is an abelian subgroup of $G$, and

(3) $\operatorname{ker}(\varphi)=\operatorname{ker}(\psi)$.

We take, as in [Chi12, Definition 1], the following

Definition 3.3. An endomorphism $\varphi$ of the group $G$ is said to be abelian is its image $G^{\varphi}$ is abelian, or equivalently $G^{\prime} \leq \operatorname{ker}(\varphi)$.

Thus all quasi-invertible fixed-point-free endomorphisms $\varphi$ of the finite group $G$ are abelian. We have in fact

Theorem 3.4. Let $G$ be a finite group, $\varphi$ a fixed-point-free endomorphism of $G$. The following are equivalent:

(1) $\varphi$ is quasi-invertible, and

(2) $\varphi$ is abelian.

We defer the proof to Section 7 . 


\section{Fitting's Lemma}

The following is a standard result in the theory of modules [Jac80, p. 113].

Theorem 4.1 (Fitting's Lemma). Let $A$ be a ring, and $M$ an $A$-module that is both artinian and noetherian.

Let $\varphi$ be an endomorphism of $M$. Then there is a a natural number $n$ such that $\operatorname{ker}\left(\varphi^{n}\right)=\operatorname{ker}\left(\varphi^{n+i}\right)$ and $M^{\varphi^{n}}=M^{\varphi^{n+i}}$ for each $i \geq 0$. We have

$$
M=\operatorname{ker}\left(\varphi^{n}\right) \oplus G^{\varphi^{n}} .
$$

Moreover, the restriction of $\varphi$ to $\operatorname{ker}\left(\varphi^{n}\right)$ is nilpotent, and the restriction of $\varphi$ to $G^{\varphi^{n}}$ is an automorphism.

The following version for groups is folklore.

Theorem 4.2 (Fitting's Lemma for groups). Let $G$ be a group which satisfies the ascending chain condition on normal subgroups, and the descending chain condition on subgroups.

Let $\varphi$ be an endomorphism of $G$. Then there is a natural number $n$ such that $\operatorname{ker}\left(\varphi^{n}\right)=\operatorname{ker}\left(\varphi^{n+i}\right)$ and $G^{\varphi^{n}}=G^{\varphi^{n+i}}$ for each $i \geq 0$. We have:

(1) $G$ is the semidirect product of the normal subgroup $K=\operatorname{ker}\left(\varphi^{n}\right)$ by the subgroup $H=G^{\varphi^{n}}$,

(2) the restriction of $\varphi$ to $K$ is nilpotent, and

(3) the restriction of $\varphi$ to $H$ is an automorphism.

We give a proof that is basically the one recorded in Car85] for finite groups.

Proof. An integer $n$ as in the statement exists because of the chain conditions. Set $H=G^{\varphi^{n}}, K=\operatorname{ker}\left(\varphi^{n}\right)$. We claim that $G$ is the semidirect product of $K$ by $H$. In fact, $K=\operatorname{ker}\left(\varphi^{n}\right)$ is normal in $G$. For each $x \in G$, we have $x^{\varphi^{n}} \in G^{\varphi^{n}}=G^{\varphi^{2 n}}$. Thus there is $y \in G$ such that $x^{\varphi^{n}}=y^{\varphi^{2 n}}$. Then

$$
\left(y^{-\varphi^{n}} x\right)^{\varphi^{n}}=y^{-\varphi^{2 n}} x^{\varphi^{n}}=1
$$

that is, $y^{-\varphi^{n}} x \in K$, so that $x=y^{\varphi^{n}}\left(y^{-\varphi^{n}} x\right) \in H K$, and $G=H K$. Now $\varphi$ is surjective on $H=G^{\varphi^{n}}=G^{\varphi^{n+1}}$. If $x \in H \cap K$, then there is $y \in G$ such that $x=y^{\varphi^{n}}$, and $y^{\varphi^{2 n}}=x^{\varphi^{n}}=1$, so that $y \in \operatorname{ker}\left(\varphi^{2 n}\right)=\operatorname{ker}\left(\varphi^{n}\right)$, and $x=1$. It follows that $H \cap K=1$, and since $\operatorname{ker}(\varphi) \leq K$, also that $\varphi$ is injective on $H$.

Proposition 3.2 yields readily

Lemma 4.3. If the fixed-point-free endomorphism $\varphi$ of the finite group $G$ has a quasi-inverse $\psi$, then $\psi$ acts on $K=\operatorname{ker}\left(\varphi^{n}\right)$ and $H=G^{\varphi^{n}}$.

Theorem 4.2 and Lemma 4.3 now yield two recipes,

(1) one for constructing all finite groups that have a quasi-invertible fixedpoint-free endomorphisms,

(2) the other for constructing all such endomorphisms for a given finite group.

The first recipe is the following.

(1) Take an arbitrary finite group $K$ and a finite abelian group $H$. 
(2) Take a fixed-point-free automorphism $\vartheta$ of $H$ (Section 51).

(3) Take a nilpotent endomorphism $\eta$ of $K$ which is abelian, that is, such that $K^{\prime} \leq \operatorname{ker}(\eta)($ Section 6)

(4) Construct a semidirect product $G$ of $K$ by $H$ such that $[K, H] \leq \operatorname{ker}(\eta)$ and $\left[H^{\vartheta}, K^{\eta}\right]=1$, and define the quasi-invertible fixed-point-free endomorphism $\varphi$ of $G$ via its restrictions $\eta$ on $K$ and $\vartheta$ on $H$ (Theorem 7.2 of Section 7).

If we are already given a finite group $G$, this reads as follows.

(1) Write $G$ as the semidirect product of a normal subgroup $K$ by an abelian subgroup $H$.

(2) Take a fixed-point-free automorphism $\vartheta$ of $H$.

(3) Take a nilpotent endomorphism $\eta$ of $K$ such that $G^{\prime}=K^{\prime}[K, H] \leq \operatorname{ker}(\eta)$ and $\left[H^{\vartheta}, K^{\eta}\right]=1$.

(4) Define the quasi-invertible fixed-point-free endomorphism $\varphi$ of $G$ via its restrictions $\eta$ on $K$ and $\vartheta$ on $H$.

\section{The ABELIAN CASE}

If $\varphi$ is a fixed-point-free endomorphism of the abelian group $G$, then $\varphi$ clearly is quasi-invertible, as noted in [Chi12, Remark 10]. In fact, $1-\varphi$ is an automorphism of $G$ here, so its inverse (with respect to map composition) is also an automorphism of $G$, and $\psi=-(1-\varphi)^{-1}+1 \in \operatorname{End}(G)$ is the quasi-inverse of $\varphi$

Since the Sylow $p$-subgroups of $G$ are fully invariant, we need only consider the case of finite abelian $p$-groups. We give a description of the fixed-point-free endomorphisms of a finite abelian $p$-group $G$, based on the approach of [HR07] to the automorphisms of a finite abelian $p$-group.

So let $G$ be a finite abelian $p$-group. Write $G$ as the direct product of homocyclic components

$$
G=H_{1} \times H_{2} \times \ldots H_{n}
$$

where each $H_{i}$ is homocyclic, of exponent $p^{e_{i}}$, with

$$
0<e_{1}<e_{2}<\cdots<e_{n} .
$$

(Clearly this decomposition is not unique in general.) If $u \in G$, we will write $u_{i}$ for the $i$-th component of $u$ with respect to the decomposition 5.1, that is, each $u_{i} \in H_{i}$ and $u=u_{1} \cdot u_{2} \cdot \ldots \cdot u_{n}$.

Let $\vartheta_{i}: H_{i} \rightarrow G$ and $\pi_{i}: G \rightarrow H_{i}$ be the injections and projections with respect to the decomposition (5.1). If $\alpha \in \operatorname{End}(G)$, write

$$
\alpha_{i j}=\vartheta_{i} \circ \alpha \circ \pi_{j}: H_{i} \rightarrow H_{j}
$$

for the $(i, j)$-th component of $\alpha$ and

$$
\beta_{i}=\left.\alpha_{i i}\right|_{\Omega_{1}\left(H_{i}\right)}
$$

for the restriction of $\alpha_{i i}$ to $\Omega_{1}\left(H_{i}\right)=\left\{x \in H_{i}: x^{p}=1\right\}$. Then we have

$$
u^{\alpha}=\prod_{i, j=1}^{n} u_{i}^{\alpha_{i j}}=\prod_{j=1}^{n}\left(\prod_{i=1}^{n} u_{i}^{\alpha_{i j}}\right) \text {, }
$$


with $\prod_{i=1}^{n} u_{i}^{\alpha_{i j}} \in H_{j}$.

Our characterization of fixed-point-free endomorphisms of $G$ is the following:

Proposition 5.1. In the above notation, the following are equivalent:

(1) $\alpha$ is a fixed-point-free endomorphism of $G$, and

(2) each $\beta_{i}$ is a fixed-point-free endomorphism of $\Omega_{1}\left(H_{i}\right)$.

Note that we can read off the action of $\beta_{i}$ also on $H_{i} / H_{i}^{p}$.

Proof. Clearly an endomorphism of $G$ has a nontrivial fixed point if and only if it has a fixed point of order $p$.

Suppose first that each $\beta_{i}$ is fixed-point-free on $\Omega_{1}\left(H_{i}\right)$. Let $u \in \Omega_{1}(G)$ be a fixed point of $\alpha$. If $i>j$, then $p^{e_{i}}=\exp \left(H_{i}\right)>\exp \left(H_{j}\right)=p^{e_{j}}$, so that $\Omega_{1}\left(H_{i}\right) \leq \operatorname{ker}\left(\alpha_{i j}\right)$. Therefore we have, according to (5.2),

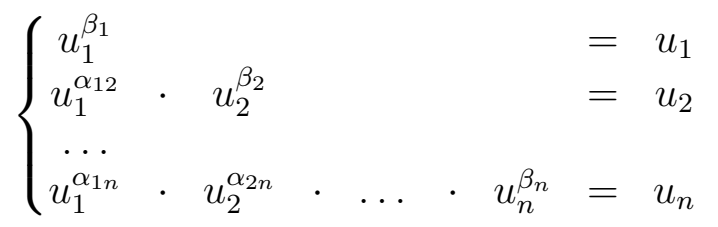

so that we have $u_{1}=0$ from the first identity, and then $u_{2}=0$ from the second one, and so on, so that $u=0$.

Conversely, suppose some of the $\beta_{i}$ have nontrivial fixed points, and let $k$ be the largest index $i$ for which this happens, so that $\beta_{k+1}, \ldots, \beta_{n}$ are fixed-point-free. We want to construct a fixed point $u$ of $\alpha$ of order $p$. We build $u$ as $u=u_{k} \cdot u_{k+1} \cdot \ldots \cdot u_{n}$, where the $u_{i} \in \Omega_{1}\left(H_{i}\right)$ are determined as follows. Here (5.3) reads

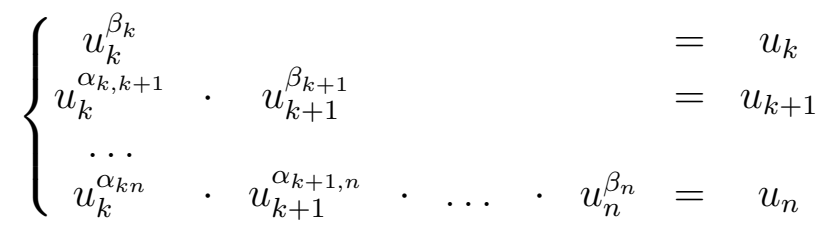

Choose $1 \neq u_{k} \in \Omega_{1}\left(H_{k}\right)$ as a nontrivial fixed point of $\beta_{k}$, so that the first equation is satisfied. Now the second equation reads

$$
u_{k}^{\alpha_{k, k+1}}=u_{k+1}^{1-\beta_{k+1}} .
$$

Since $\beta_{k+1}$ is fixed-point-free on $\Omega_{1}\left(H_{k+1}\right)$, the function $1-\beta_{k+1}$ is bijective on $\Omega_{1}\left(H_{k+1}\right)$ by Proposition 2.3, so that there is a $u_{k+1} \in \Omega_{1}\left(H_{k+1}\right)$ which fulfills (5.5)). Proceeding in the same fashion, we find values for $u_{k+2}, \ldots, u_{n}$ that satisfy all the equations of (5.4), so that the resulting $u$ is a nontrivial fixed point of $\varphi$ of order $p$.

\section{Nilpotent ENDOMORPhisms}

We record a couple of immediate facts here.

Proposition 6.1. Let $G$ be a finite group, $\varphi$ a nilpotent endomorphism of $G$.

Then $\varphi$ is fixed-point-free. Moreover, the following are equivalent

(1) $\varphi$ is quasi-invertible, and

(2) $\varphi$ is abelian. 
Proof. If $\varphi^{n}=0$, and $x=x^{\varphi}$ for some $x \in G$, then $x=x^{\varphi^{n}}=1$.

If $\varphi$ is quasi-invertible, then $G^{\varphi}$ is abelian by Proposition 3.2.

Conversely, let $G^{\varphi}$ be abelian. The inverse (with respect to map composition) of the map $1-\varphi$ is clearly $1+\varphi+\varphi^{2}+\cdots+\varphi^{n-1}$, so if $\varphi$ has a quasi-inverse $\psi$, then this is $\psi=-\left(\varphi+\varphi^{2}+\cdots+\varphi^{n-1}\right)$. But $\psi$ is the composition of $\varphi$ with the restriction of $-\left(1+\varphi+\cdots+\varphi^{n-2}\right)$ to $G^{\varphi}$. Since the latter group is abelian, $-\left(1+\varphi+\cdots+\varphi^{n-2}\right)$ is an endomorphism of $G^{\varphi}$ by Proposition [2.1, so that $\psi$ is an endomorphism of $G$, and thus $\psi$ is indeed the quasi-inverse of $\varphi$.

\section{Piecing Endomorphisms together}

Let $G$ be the semidirect product of the normal subgroup $K$ by the subgroup $H$. Let $\vartheta \in \operatorname{End}(H), \eta \in \operatorname{End}(K)$. We define a map $\varphi$ on $G$, the semidirect product of $K$ by $H$, by letting

$$
(h k)^{\varphi}=h^{\vartheta} k^{\eta},
$$

for $h \in H$ and $k \in K$. This will be an endomorphism of $G$ is and only if for all $h, h^{\prime} \in H$ and $k, k^{\prime} \in K$

$$
\left(h^{\prime} k h k^{\prime}\right)^{\varphi}=\left(h^{\prime} h k^{h} k^{\prime}\right)^{\varphi}=h^{\prime \vartheta} h^{\vartheta}\left(k^{h}\right)^{\eta} k^{\prime \eta}
$$

equals

$$
\left(h^{\prime} k\right)^{\varphi}\left(h k^{\prime}\right)^{\varphi}=h^{\prime \vartheta} k^{\eta} h^{\vartheta} k^{\prime \eta} .
$$

This means

$$
\left(k^{h}\right)^{\eta}=\left(k^{\eta}\right)^{h^{\vartheta}},
$$

for all $h \in H$ and $k \in K$, that is, $\eta: K \rightarrow K$ is a twisted morphism of $H$-modules, or equivalently, multiplying both sides of the previous equations on the left by $k^{-\eta}$

$$
[k, h]^{\eta}=\left[k^{\eta}, h^{\vartheta}\right]
$$

for all $h \in H$ and $k \in K$. Now we note

Lemma 7.1. If $\varphi$ is an abelian fixed-point-free endomorphism of the finite group $G$, then both terms of (7.2) vanish for all $h \in H$ and $k \in K$.

Proof. The right-hand term vanishes because $G^{\varphi}$ is abelian and contains $H^{\vartheta}$ and $K^{\eta}$, and the left one vanishes since $[K, H] \leq G^{\prime} \cap K \leq \operatorname{ker}(\varphi) \cap K \leq \operatorname{ker}(\eta)$, again because $G^{\varphi}$ is abelian.

We may state

Theorem 7.2. Let the finite group $G$ be the semidirect product of the group $K$ by the abelian group $H$. Let $\vartheta$ be a fixed-point-free endomorphism of $H$, and $\eta$ be a nilpotent endomorphism of $K$. Define a map $\varphi$ on $G$ by

$$
(h k)^{\varphi}=h^{\vartheta} k^{\eta} \text {, }
$$

for $h \in H$ and $k \in K$.

Then the following are equivalent

(1) $\varphi$ is a quasi-invertible fixed-point-free endomorphism, and

(2) $G^{\prime}=K^{\prime}[K, H] \leq \operatorname{ker}(\eta)$, and $\left[K^{\eta}, H^{\vartheta}\right]=1$. 
Note that the condition $G^{\prime} \leq \operatorname{ker}(\eta)$ contains both the condition $[K, H] \leq$ $\operatorname{ker}(\eta)$, that we have seen to be necessary for $\varphi$ to be an abelian endomorphism, and the condition $K^{\prime} \leq \operatorname{ker}(\eta)$ which states that $\eta$ is an abelian endomorphism.

Proof. We are only left with proving that $\varphi$ has a quasi-inverse under the hypotheses on $\eta, \vartheta$, and the conditions of (2). Now by the results of Sections 5 and 6 , the endomorphisms $\eta$ and $\vartheta$ have quasi-inverses $\eta^{\prime}$ and $\vartheta^{\prime}$. By the conditions of (2), and Proposition 3.2 , we have $[K, H] \leq \operatorname{ker}(\eta)=\operatorname{ker}\left(\eta^{\prime}\right)$ and $\left[K^{\eta^{\prime}}, H^{\vartheta^{\prime}}\right]=\left[K^{\eta}, H^{\vartheta}\right]=1$, so that the conditions for $\eta^{\prime}$ and $\vartheta^{\prime}$ to induce an endomorphism $\psi$ of $G$ are satisfied. This will be the quasi-inverse of $\varphi$, as for $h \in H$ and $k \in K$ we have

$$
(h k)^{-\varphi+\varphi \psi-\psi}=(h k)^{-\varphi}(h k)^{\varphi \psi}(h k)^{-\psi}=k^{-\eta} h^{-\vartheta} h^{\vartheta \vartheta^{\prime}} k^{\eta \eta^{\prime}} k^{-\eta^{\prime}} h^{-\vartheta^{\prime}} .
$$

Now all terms commute, as $K^{\eta}=K^{\eta^{\prime}}, H^{\vartheta}=H^{\vartheta^{\prime}}$ are abelian and $\left[K^{\eta}, H^{\vartheta}\right]=1$. So we have

$$
(h k)^{-\varphi+\varphi \psi-\psi}=h^{-\vartheta} h^{\vartheta \vartheta^{\prime}} h^{-\vartheta^{\prime}} k^{-\eta} k^{\eta \eta^{\prime}} k^{-\eta^{\prime}}=h^{-\vartheta+\vartheta \vartheta^{\prime}-\vartheta^{\prime}} k^{-\eta+\eta \eta^{\prime}-\eta^{\prime}}=1,
$$

that is, $\psi$ is the quasi-inverse of $\varphi$.

We are now in a position to give the

Proof of Theorem 3.4. Let $\varphi$ be an abelian fixed-point-free endomorphism of the group $G$. Decompose $G$ as in Theorem 4.2, and let $\eta$ be the restriction of $\varphi$ to $K$, and $\vartheta$ the restriction of $\varphi$ to H. By Lemma 7.1, and the fact that $\varphi$ is abelian, conditions (2) of Theorem 7.2 are satisfied, so that $\varphi$ has a quasi-inverse.

\section{EXAMPLES}

We review first the examples of [Chi12, Section 5] from the point of view of this paper.

One of the examples is a Frobenius group (see [Gor68, 4.5], [Isa76, Chap. 7]) $G$ with Frobenius kernel $A$ and cyclic complement. (The kernel is taken to be abelian in the original example, but this is immaterial.)

So we have $A=G^{\prime}$, and $(|A|,|G: A|)=1$. Let $\varphi$ be a nontrivial fixed-pointfree endomorphism of $G$. We have $A=G^{\prime} \leq \operatorname{ker}(\varphi)$ by Proposition 3.2. Choose a Frobenius complement $\langle b\rangle$ such that $G^{\varphi} \leq\langle b\rangle$, so that $\langle b\rangle$ is $\varphi$-invariant. We are left with determining the fixed-point-free endomorphisms of $\langle b\rangle$. These are, as noted in [Chi12], the maps $b \mapsto b^{s}$, with $(s-1,|b|)=1$. Proposition 5.1 yields the equivalent condition $s \not \equiv 1(\bmod p)$, for all primes dividing $|b|$.

Clearly a group of order 2 has no nontrivial fixed-point-free automorphisms. Thus the subgroup $K$ of Section 4 cannot have index 2 in the group $G$.

This situation occurs in Chi12 first when $G=S_{n}$, the symmetric group on $n \geq 3$ letters. Here $S_{n}^{\prime}=A_{n} \leq K$, so we conclude as in [Chi12] that $K=S_{n}$, and a nontrivial abelian fixed-point-free endomorphism must be nilpotent, and thus map $G$ onto a subgroup generated by an even involution.

The case of dihedral groups is also described in [Chi12, Section 5]; we review it here according to our approach. 
Let $G$ be a dihedral group, $\varphi$ be a nontrivial abelian fixed-point-free endomorphism of $G$, and $H$ and $K$ be the subgroups of Section 4. Fix an element $x$ such that $\langle x\rangle$ has index 2 in $G$, and an involution $y \notin\langle x\rangle$.

If $G$ has order twice an odd number, then $G^{\prime}=\langle x\rangle$ has index 2. Since $K \geq$ $\operatorname{ker}(\varphi)=G^{\prime}$, by the above argument $\varphi$ should nilpotent, with $G^{\varphi}$ a subgroup of order 2 of $\operatorname{ker}(\varphi)=\langle x\rangle$. But the latter is a group of odd order, so there are no abelian fixed-point-free endomorphisms in this case.

If $G$ has order $4 m$, then $G^{\prime}=\left\langle x^{2}\right\rangle$ has order $m$. We first discuss what $K \geq G^{\prime}$ can be.

If $K=G^{\prime}$, then $G^{\prime}$ must have a complement $H$. This occurs if and only if $m$ is odd, and then $H$ is one of the Klein four-groups $\left\langle x^{m}, x^{i} y\right\rangle$. We obtain $\varphi$ by extending the identity on $K$ by a (fixed-point-free) automorphism of order 3 of $H$. This covers case (5) with $i$ odd, and case (7) with $i$ even of [Chi12, Section 5].

If $K>G^{\prime}$, then $K$ cannot have index 2 , as noted above, so that $K=G$, and $\varphi$ is nilpotent. We discuss the possibilities for $\operatorname{ker}(\varphi) \geq G^{\prime}$.

If $\operatorname{ker}(\varphi)=G^{\prime}$, then $G^{\varphi}$ is one of the Klein four-groups $\left\langle x^{m}, x^{i} y\right\rangle$. These have to intersect $\operatorname{ker}(\varphi)=\left\langle x^{2}\right\rangle$ in $\left\langle x^{m}\right\rangle$, and then $m$ must be even: this covers case (5) with $i$ even, and case (7) with $i$ odd.

If $\operatorname{ker}(\varphi)>G^{\prime}$, there are three possibilities for the maximal subgroup $\operatorname{ker}(\varphi)$ of $G$. (Here $G^{\varphi}$ is a subgroup of order 2 of $\operatorname{ker}(\varphi)$.)

(1) If $\operatorname{ker}(\varphi)=\langle x\rangle$, then $G^{\varphi}=\left\langle x^{m}\right\rangle$, and we get case (1).

(2) If $\operatorname{ker}(\varphi)=\left\langle x^{2}, y\right\rangle$, then either $m$ is even and $G^{\varphi}=\left\langle x^{m}\right\rangle$ (case (3)), or $G^{\varphi}=\left\langle x^{2 a} y\right\rangle$ for some $a$ (case (4)).

(3) If $\operatorname{ker}(\varphi)=\left\langle x^{2}, x y\right\rangle$, then either $m$ is even and $G^{\varphi}=\left\langle x^{m}\right\rangle$ (case (2)), or $G^{\varphi}=\left\langle x^{2 a+1} y\right\rangle$ for some $a($ case $(6))$.

We now give a couple more examples.

Consider a semidirect product $G=H K$, with $K$ normal in $G$, and $H$ abelian. Clearly all fixed-point-free endomorphisms of $H$ induce abelian fixed-point-free endomorphisms $\varphi$ of $G$ with $K \leq \operatorname{ker}(\varphi)$. In all the examples so far, and in the notation of Theorem 4.2, we have had either $K=G$ (that is, $\varphi$ is nilpotent), or $\varphi$ trivial on $K$. We now give an example in which $K<G$, and $\varphi$ acts nontrivially on $K$.

Let $K$ be a (nonabelian) special $p$-group [Hup67, III.13], that is, a group in which $K^{\prime}=Z(K)=\operatorname{Frat}(K)$ is elementary abelian. (Here Frat $(K)=K^{\prime} K^{p}$ is the Frattini subgroup of $K$.) The endomorphisms in $\operatorname{Hom}(K, Z(K)) \cong \operatorname{Hom}\left(K / K^{\prime}, Z(K)\right)$ are then all nilpotent. Consider the elementary abelian group $H$ of central automorphisms of $K$,

$$
H=\{1+f: f \in \operatorname{Hom}(K, Z(K))\},
$$

and let $G$ be the natural extension of $K$ by $H$. Define $\eta$ on $K$ to be any element of $\operatorname{Hom}(K, Z(K))$, and $\vartheta$ to be any fixed-point-free endomorphism of $H$. (We might for instance regard the elementary abelian group $H$ as the additive group of a finite field $E$, and take $\vartheta$ to be the multiplication by an element of $E$ different from 0,1 .) 
Clearly $[K, H]=Z(K) \leq \operatorname{ker}(\eta)$, and $K^{\eta} \leq Z(K)$ commutes with $H^{\vartheta} \leq H$. The recipe of Section 5 is thus satisfied, so that this defines a quasi-invertible fixed-point-free endomorphism of $G$.

However, Childs shows in [Chi12, Theorem 2] that two fixed-point-free endomorphisms $\varphi, \psi$ of the finite group $G$ induce the same regular subgroup of the group of permutations on $G$ (as in the Introduction) if and only if there is a fixed-point-free endomorphim $\zeta: G \rightarrow G$, with $G^{\zeta} \leq Z(G)$, such that $1-\varphi=(1-\zeta)(1-\psi)$, or $\varphi=\psi-\zeta \psi+\zeta$. We say that two abelian endomorphisms fixed-point-free $\varphi, \psi$ are equivalent if they satisfy this condition.

Now it is not difficult to see that in the last example $\varphi$ is equivalent in this sense to another abelian fixed-point-free endomorphism which acts trivially on $K$.

To see an example where this does not happen, take $K$ to be the nonabelian $p$-group of order $p^{3}$ and exponent $p$, for $p$ odd. If $K=\langle a, b\rangle$, let $\eta$ be the nilpotent endomorphism of $K$ defined by $a \mapsto b \mapsto 1$, so $K^{\eta}=\langle b\rangle$ is abelian, but not central in $K$. We may then consider for instance the automorphism $\alpha$ of $K$ of order $p$ which acts as $a \mapsto a b, b \mapsto b$, and let $H=\langle\alpha\rangle$. For $\vartheta$ we may take any fixedpoint-free endomorphism of $H$, that is, any map $\alpha \mapsto \alpha^{s}$, with $s \not \equiv 1(\bmod p)$. If $G=H K$, then we have $G^{\prime}=\langle b, z\rangle \leq \operatorname{ker}(\eta)$, and $\left[K^{\eta}, H^{\vartheta}\right] \leq[\langle b\rangle, H]=1$, so that the recipe of Section 5 is satisfied.

\section{REFERENCES}

[By96a] N. P. Byott, Uniqueness of Hopf Galois structure for separable field extensions, Comm. Algebra 24 (1996), no. 10, 3217-3228. MR 1402555 (97j:16051a)

[By96b] N. P. Byott, Corrigendum: "Uniqueness of Hopf Galois structure for separable field extensions", Comm. Algebra 24 (1996), no. 11, 3705. MR 1405283 (97j:16051b)

[Car85] A. Caranti, Finite p-groups of exponent $p^{2}$ in which each element commutes with its endomorphic images, J. Algebra 97 (1985), no. 1, 1-13. MR 812164 (87b:20029)

[CC07] Lindsay N. Childs and Jesse Corradino, Cayley's theorem and Hopf Galois structures for semidirect products of cyclic groups, J. Algebra 308 (2007), no. 1, 236-251. MR 2290920 (2007j:20026)

[Chi00] Lindsay N. Childs, Taming wild extensions: Hopf algebras and local Galois module theory, Mathematical Surveys and Monographs, vol. 80, American Mathematical Society, Providence, RI, 2000. MR 1767499 (2001e:11116)

[Chi07] _ Some Hopf Galois structures arising from elementary abelian p-groups, Proc. Amer. Math. Soc. 135 (2007), no. 11, 3453-3460. MR 2336557 (2008j:16107)

[Chi12] Lindsay N. Childs, Fixed-point free endomorphisms and Hopf Galois structures, Proc. Amer. Math. Soc. (2012).

[Gor68] Daniel Gorenstein, Finite groups, Harper \& Row Publishers, New York, 1968. MR 0231903 (38 \#229)

[GP87] Cornelius Greither and Bodo Pareigis, Hopf Galois theory for separable field extensions, J. Algebra 106 (1987), no. 1, 239-258. MR 878476 (88i:12006)

[HR07] Christopher J. Hillar and Darren L. Rhea, Automorphisms of finite abelian groups, Amer. Math. Monthly 114 (2007), no. 10, 917-923. MR 2363058

[Hup67] B. Huppert, Endliche Gruppen. I, Die Grundlehren der Mathematischen Wissenschaften, Band 134, Springer-Verlag, Berlin, 1967. MR 0224703 (37 \#302)

[Isa76] I. Martin Isaacs, Character theory of finite groups, Academic Press [Harcourt Brace Jovanovich Publishers], New York, 1976, Pure and Applied Mathematics, No. 69. MR 0460423 (57 \#417) 
[Jac64] Nathan Jacobson, Structure of rings, American Mathematical Society Colloquium Publications, Vol. 37. Revised edition, American Mathematical Society, Providence, R.I., 1964. MR 0222106 (36 \#5158)

[Jac80] _ Basic algebra. II, W. H. Freeman and Co., San Francisco, Calif., 1980. MR 571884 (81g:00001)

[Pil83] Günter Pilz, Near-rings, second ed., North-Holland Mathematics Studies, vol. 23, NorthHolland Publishing Co., Amsterdam, 1983, The theory and its applications. MR 721171 (85h:16046)

(A. Caranti) Dipartimento di Matematica, Universitì degli Studi di Trento, via Sommarive 14, I-38123 Trento, Italy

E-mail address: andrea.caranti@unitn.it

$U R L:$ http://science.unitn.it/ caranti/ 